\title{
Use of intermolecular distances from ssNMR in crystal structure determination from powder diffraction data
}

\author{
Jan Rohlíček ${ }^{1}$, Libor Kobera², Michal Hušák ${ }^{3}$, Jiří Brus² \\ ${ }^{1}$ Institute of Physics of the Czech Academy of Sciences, Na Slovance 2, Prague 8, 182 21, Czech Republic; \\ ${ }_{2}^{2}$ Institute of Macromolecular Chemistry, Czech Academy of Sciences, Heyrovsky Square 2, Prague, 16206, Czech Republic; \\ ${ }^{3}$ Department of Solid State Chemistry, University of Chemical Technology Prague, Prague 6, 166 28, Czech Republic;
}

$$
\text { rohlicek@fzu.cz }
$$

The steady-state in the field of software allowing the application of different approaches for crystal structure determination $[1,2]$ could give the impression that the determination of the crystal structure from the powder diffraction data is a common and straightforward task that does not deserve additional attention. However, looking at crystal structures determined from powder diffraction data, one can estimate the limit of the method. In the words of degrees of freedom, the current limit is around 40. The two most complex crystal structures found in the CSD database [3, 4], and also many others, were solved using the direct-space methods [5]. Simply said, the direct-space methods find the structural model by changing the position and shape of the molecular fragments in the asymmetric part of the unit cell. They allow defining additional conditions for the studied crystal structure, which is handled as additional observation. For example, the model can be restricted by several specifying torsion angles or by rigid groups as it is already implemented in existing software [6,7]. All these additional observations aim to make it possible to find a solution or at least significantly reduce the calculation time.

Another observation that may increase the probability of finding the correct solution is the information about intermolecular distances in the crystal structure. This information can be obtained by performing a specific ssNMR measurement which usually offers a list of short-range interactions between atoms. We decided to implement such a possibility to the already existing software FOX [5], and we tested it on several compounds. First of all, we tested it on the already solved crystal structures. We defined intermolecular distances between several selected atoms with various precisions, and we used them as additional restrictions that influenced the final cost function. We then tested it on a compound with an unknown crystal structure, for which we obtained estimated intermolecular distances from the ssNMR. We used these additional observations for the structure solution process from X-ray powder diffraction data.

[1] David, W.I.F.; Shankland, K. Structure Determination from Powder Diffraction Data. Acta Crystallogr. A 2008, 64, 52-64, doi:10.1107/S0108767307064252.

[2] Meden, A.; Radosavljevic Evans, I. Structure Determination from Powder Diffraction Data: Past, Present and Future Challenges: Structure Determination from Powder Diffraction Data: Past, Present and Future. Cryst. Res. Technol. 2015, 50, 747-758, doi:10.1002/crat.201500048.

[3] Husak, M.; Jegorov, A.; Czernek, J.; Rohlicek, J.; Zizkova, S.; Vraspir, P.; Kolesa, P.; Fitch, A.; Brus, J. Successful Strategy for High Degree of Freedom Crystal Structure Determination from Powder X-Ray Diffraction Data: A Case Study for Selexipag Form I with 38 DOF. Cryst. GROWTH Des. 2019, 19, 4625-4631, doi:10.1021/acs.cgd.9b00517.

[4] Fernandes, P.; Shankland, K.; Florence, A.J.; Shankland, N.; Johnston, A. Solving Molecular Crystal Structures from X-Ray Powder Diffraction Data: The Challenges Posed by $\gamma$-Carbamazepine and Chlorothiazide N,N,-Dimethylformamide (1/2) Solvate. J. Pharm. Sci. 2007, 96, 1192-1202, doi:10.1002/jps.20942.

[5] Černý, R.; Favre-Nicolin, V. Direct Space Methods of Structure Determination from Powder Diffraction: Principles, Guidelines and Perspectives. Z. Für Krist. - Cryst. Mater. 2007, 222, doi:10.1524/zkri.2007.222.3-4.105.

[6] Černý, R.; Favre-Nicolin, V.; Rohlíček, J.; Hušák, M. FOX, Current State and Possibilities. Crystals 2017, 7, 322, doi:10.3390/cryst7100322.

[7] David, W.I.F.; Shankland, K.; van de Streek, J.; Pidcock, E.; Motherwell, W.D.S.; Cole, J.C. DASH: A Program for Crystal Structure Determination from Powder Diffraction Data. J. Appl. Crystallogr. 2006, 39, 910-915, doi:10.1107/S0021889806042117.

Keywords: Powder diffraction; Direct-space methods; ssNMR; ab-initio; structure solution 